\title{
A seven-miRNA expression-based prognostic signature and its corresponding potential competing endogenous RNA network in early pancreatic cancer
}

\author{
XUE BAI ${ }^{1 *}$, DONGLAN LU ${ }^{1 *}$, YAN LIN ${ }^{2}$, YUFENG LV ${ }^{3}$ and LIUSHENG HE ${ }^{4}$ \\ ${ }^{1}$ Department of Medical Oncology, The Second Affiliated Hospital of Guilin Medical University, Guilin, Guangxi 541199; \\ ${ }^{2}$ Department of Medical Oncology, Affiliated Tumor Hospital of Guangxi Medical University, Nanning, Guangxi 530021; \\ ${ }^{3}$ Department of Medical Oncology, Affiliated Langdong Hospital of Guangxi Medical University, Nanning, Guangxi 530029; \\ ${ }^{4}$ Department of Surgery 1, Minzu Hospital of Guangxi Zhuang Autonomous Region, Nanning, Guangxi 530001, P.R. China
}

Received January 29, 2018; Accepted May 23, 2019

DOI: $10.3892 / \mathrm{etm} .2019 .7728$

\begin{abstract}
The present study aimed to establish a microRNA (miRNA/miR) signature to predict the prognosis of patients with pancreatic cancer (PC) at the early stage and to investigate the involvement of competing endogenous RNAs (ceRNAs) in PC. Using mature miRNA expression profiles from The Cancer Genome Atlas, differentially expressed miRNAs in tissues derived from patients exhibiting early PC and tissues from healthy individuals were compared. The least absolute shrinkage and selection operator regression method was used to construct a miRNA-based signature for predicting prognosis. The miRNet tool, gene set enrichment analysis (GSEA) and the LncRNADisease database were utilized to explore the mechanistic involvement of ceRNAs. A total of seven downregulated miRNAs in PC (miR-424-5p, miR-139-5p, miR-5586-5p, miR-126-3p, miR-3613-5p, miR-454-3p and miR-1271-5p) were selected to generate a signature. Based on this seven-miRNA signature, it was possible to stratify patients with PC into low- and high-risk groups. The overall survival of the low-risk group was significantly longer than that of the high-risk group $(\mathrm{P}<0.001)$. The seven-miRNA signature was able to predict the 2-year-survival rate of patients with early PC with an area under the curve of 0.750 . Furthermore, as opposed
\end{abstract}

Correspondence to: Dr Liusheng He, Department of Surgery 1, Minzu Hospital of Guangxi Zhuang Autonomous Region, 232 Mingxiu Road, Nanning, Guangxi 530001, P.R. China

E-mail: liusheng_he@126.com

Dr Yufeng Lv, Department of Medical Oncology, Affiliated Langdong Hospital of Guangxi Medical University, 60 Xiangbin Road, Nanning, Guangxi 530029, P.R. China

E-mail: tougaozhuanyong_lv@126.com

*Contributed equally

Key words: pancreatic cancer, microRNA signature, prognosis, competing endogenous RNA to routine clinicopathological features, this seven-miRNA signature was an independent prognostic factor according to multivariate Cox regression analysis. GSEA indicated that the extracellular matrix receptor interaction pathway and the transforming growth factor- $\beta$ signaling pathway were enriched in the high-risk group. A ceRNA network of the seven-miR signature was constructed. In conclusion, the present study provided a seven-miRNA signature, according to which patients with early PC may be divided into high- and low-risk groups. The ceRNA network of the prognostic signature was preliminarily explored.

\section{Introduction}

Pancreatic cancer (PC), with almost as many deaths $(n=432,000)$ as cases $(n=459,000)$, is a highly lethal malignancy and the seventh leading cause of cancer-associated mortality in 2018 worldwide (1). Indeed, the incidence of PC continues to rise, and it is predicted to become the second leading cause of cancer-associated mortality by the year 2030 in the United States (2). The poor prognosis of PC is attributed to its asymptomatic nature until the late stages and invasive features, with a $<20 \%$ chance of being operable at the time of diagnosis (3). Furthermore, $>50 \%$ of cases of early PC after surgery encounter recurrences within 12 months (4), with a 5 -year survival rate of up to $45 \%$ after curative surgery (5). The poor prognosis of $\mathrm{PC}$ is due to most cases of PC being diagnosed at the late stage when they have missed the time window for curative surgery (3) and the fact that its molecular pathogenesis remains largely elusive. To improve outcomes for patients with $\mathrm{PC}$, investigation into prognostic biomarkers and their molecular mechanisms in the early stages of disease is imperative to prolong patient survival. Using reliable biomarkers, high-risk patients may be followed up more frequently after surgery instead of being dictated by the routine schedule.

MicroRNAs (miRNAs/miRs), a class of short RNAs (6), are promising prognostic predictors for various types of cancer (7-9), including PC (7). miRNAs have crucial roles in transcriptional regulation of gene expression via 
several mechanisms (10). Competing endogenous RNAs (ceRNAs) constitute one of these mechanisms. This type of RNA crosstalk exists between protein-coding mRNAs and non-coding RNAs, including miRNAs and long non-coding RNAs (lncRNAs) (11-13). Various studies have proposed miRNA-based signatures for prognosis prediction for PC (14-16); however, few of them focused on early PC and corresponding potential ceRNA networks.

In the present study, the mature miRNA expression profiles and clinical information of cases of early PC in The Cancer Genome Atlas (TCGA) database were comprehensively analyzed using Bioinformatics. To the best of our knowledge, the present study is the first to not only propose a novel seven-miRNA prognostic signature, but also predict a ceRNA network for early PC.

\section{Materials and methods}

Data processing. The pre-processed mature miRNA expression profiles for PC and pancreatic tissues from healthy individuals in The Cancer Genome Atlas (TCGA) database (https://www.cancer. gov/about-nci/organization/ccg/research/structural-genomics/tcga/usingtcga/citing-tcga), displayed as log2-converted reads per million $[\log 2(\mathrm{RPM}+1)]$, were downloaded from the University of California Santa Cruz Xena database (17). The mature miRNA expression profiles covered 2,050 miRNAs. Corresponding clinical information was downloaded from the TCGA database (accession date, 10/14/2018). The mature miRNA expression profiles contained 182 samples (178 PC tissues and four pancreatic tissues from healthy individuals) based on the IlluminaHiSeq_miRNASeq platform (Illumina Inc.). The inclusion criteria for screening differentially expressed miRNAs were as follows: i) The dataset included miRNA expression profiles; ii) the samples were from patients with stage IA-IIB disease according to the American Joint Committee on Cancer Tumor-Node-Metastasis (TNM) system (https://cancerstaging. org/) (18), and iii) the miRNAs were expressed in $>20 \%$ of samples of PC. The data for a total of 167 samples of PC with stage IA-IIB disease and four matched healthy pancreatic tissues were extracted for screening differentially expressed miRNAs. As all of the data were publicly available and open-access, ethical approval was not required, and the study adhered to the TCGA publication guidelines and data access policies (https://cancergenome.nih.gov/).

Screening of differentially expressed miRNAs. The differentially expressed miRNAs between PCs and pancreatic tissues from healthy individuals were analyzed using the 'limma' package (19) in R. The fold changes (FCs) in the expression of individual miRNAs were calculated and differentially expressed miRs with $|\log 2 \mathrm{FCl}|>1$ and $\mathrm{P}<0.05$ adjusted by the false discovery rate (FDR) were considered as significant $(8,9,20)$.

Identification of the miRNA-based prognostic signature for early $P C$. The expression values of differentially expressed miRNAs in each sample of PC were extracted. Patients with a survival time of $<30$ days from the day of diagnosis were removed and 161 patients remained for survival analysis. The method of least absolute shrinkage and selection operator (LASSO) for regression may be used for optimal selection of features in high-dimensional data with a strong predictive value and low correlation between one another to prevent overfitting (21). This approach may be applied to the Cox proportional hazard regression model for survival analysis with high-dimensional data (22). Therefore, as in a previous high-quality study (23), the LASSO Cox regression model was used to select the most useful predictive markers among all differentially expressed miRNAs, and a multi-miRNA-based signature was constructed for predicting prognosis. The analysis was performed using the 'glmnet' package (https://CRAN.R-project.org/package=glmnet) in R. A risk score was created using the regression coefficients from the LASSO analysis to weight the expression value of the selected miRNAs with the following formula:

Prognostic Index $(\mathrm{PI})=\operatorname{expr}_{\text {miRnA1 }} \mathrm{x} \mathrm{Coef}_{1}+\operatorname{expr}_{\text {miRnA2 }} \mathrm{x} \mathrm{Coef}_{2}$ $+\operatorname{expr}_{\text {miRnA3 }} \times \mathrm{Coef}_{3}+\ldots$

Where the 'Coef' value is the estimated regression coefficient of a certain miRNA and is derived from the LASSO Cox regression analysis, and 'expr' indicates the expression value of the miRNAs. Patients with early PC were divided into low-risk and high-risk groups, according to the median PI. Time-dependent receiver operating characteristic (ROC) analysis was used to assess the predictive value of the miR-based signature for 2-year-survival of PC, performed with the 'survivalROC' package in R (24).

Prediction of the target genes of the prognostic miRNA-based signature and pathway enrichment analysis. The miRNet tool integrates data from 11 different miRNA databases (25) (http://www.mirnet.ca/). Using miRNet, the targets (mRNAs and IncRNAs) of the prognostic miRNA-based signature were predicted and pathway enrichment analysis of the target mRNAs was performed. A lncRNA-miRNA-gene network was constructed. The network was further optimized to improve visualization using Cytoscape software (26).

Gene set enrichment analysis (GSEA) and construction of ceRNA network of the miRNA-based signature. Given that a single miRNA is able to modulate the levels of several hundreds of target mRNAs and affect a myriad of cellular processes, it is promising to explore the ultimate effects of the interactions of these cellular processes that these seven miRNAs are implicated in. The normalized fragments per kilobase of transcript per million mapped reads values from the RNA-sequencing data of the corresponding 161 patients with early PC were obtained from TCGA Data Portal (https://portal.gdc.cancer.gov/). GSEA was performed by the JAVA program (http://www.broadinstitute.org/gsea) using the MSigDB c2.cp.kegg.v6.2.symbols.gmt gene set collection. Gene sets with FDR of $<0.25$ after performing 1,000 permutations were considered to be significantly enriched (27). The LncRNADisease database (www.cuilab.cn/lncrnadisease) is a resource that curates the experimentally supported lncRNA-disease association data (28). The PC-associated lncRNAs were obtained from the lncRNADisease database. The common lncRNAs between target lncRNAs predicted by miRNet and the PC-associated IncRNAs, and the common 
A

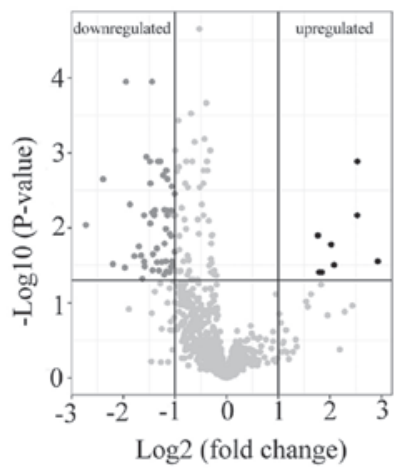

$\mathrm{C}$

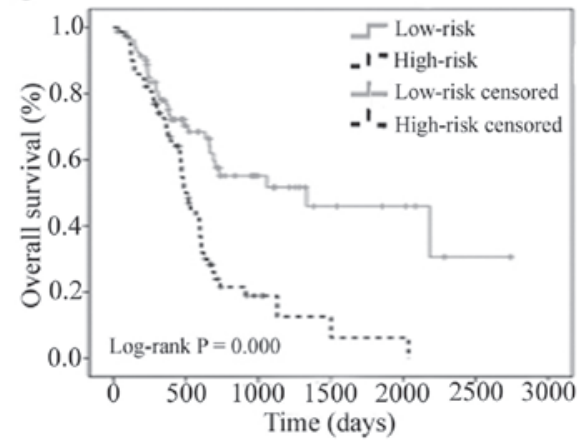

B

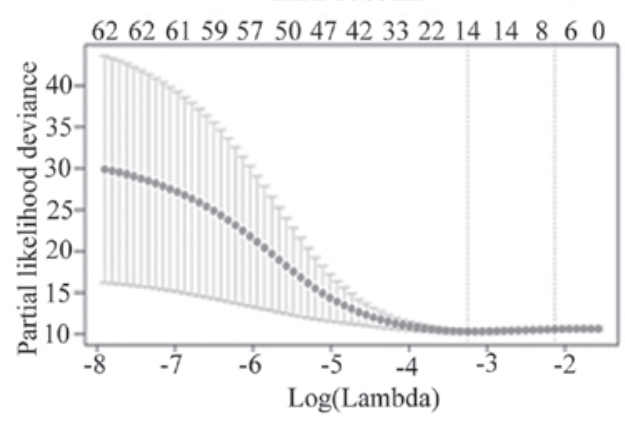

$\mathrm{D}$

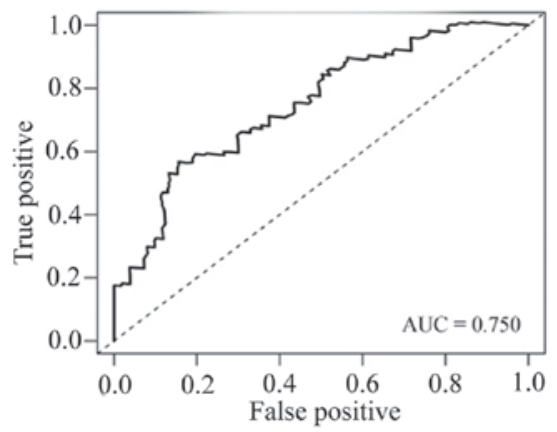

Figure 1. (A) Volcano plot of the differentially expressed miRNAs between pancreatic cancer and healthy pancreatic tissue. (B) 20 -fold cross-validation for tuning parameter selection in the least absolute shrinkage and selection operator model. (C) Kaplan-Meier survival curve analysis of the seven-microRNA signature prognostic index. (D) Receiver operating characteristic curve analysis of the predictive value of the seven-microRNA signature as a prognostic index for the 2-year-survival rate. miRNA, microRNA; AUC, area under curve.

genes between target genes predicted by miRNet and the core enrichment genes provided by GSEA were extracted. The ceRNA network of the miR-based signature in early PC was constructed using Cytoscape software.

Statistical analysis. The unpaired t-test was used to screen differentially expressed miRNAs. Univariate/multivariate Cox proportional hazards analyses and Kaplan-Meier survival analysis with log-rank test were used to compare survival between the two groups of patients. The survival analysis was performed using SPSS statistics software version 22.0 (IBM Corp.). All tests were two-sided and $\mathrm{P}<0.05$ was considered to indicate statistical significance.

\section{Results}

Differentially expressed miRNAs between early PC and pancreatic tissues from healthy individuals. The detailed clinical characteristics of the patients with PC at the early stage, including sex, age at diagnosis and TNM stage are listed in Table I. According to the cutoff criteria $(\mathrm{P}<0.05$ and $\mid \log 2 \mathrm{FCl}>1), 62$ miRNAs were identified to be differentially expressed between PC and pancreatic tissues from healthy individuals. These include eight miRNAs that were upregulated and 54 miRNAs that were downregulated in PC tissues. The results of the expression analysis are presented as a volcano plot (Fig. 1A) to demonstrate that the distribution of P-values and $\log 2 \mathrm{FCl}$ was reasonable with respect to each other.

Identification of seven-miRNA prognostic signature for early $P C$. In order to develop a miRNA-based signature for predicting prognosis in early PC, LASSO Cox regression was performed using the expression profiles of the 62 differentially expressed miRNAs. Using the LASSO method and 20-fold cross-validation, seven miRNAs were identified with non-zero regression coefficients (Fig. 1B). A risk score was created by establishing the following formula:

$\mathrm{PI}=\exp _{\mathrm{miR}-424-5 \mathrm{p}} \times 0.111+\exp _{\mathrm{miR}-139-5 \mathrm{p}} \times(-0.029)+\exp _{\mathrm{miR}-5586-5 \mathrm{p}}$ $\mathrm{x}(-0.070)+\exp _{\mathrm{miR}-126-3 \mathrm{p}} \times(-0.026)+\exp _{\mathrm{miR}-3613-5 \mathrm{p}} \times(-0.199)+$ $\exp _{\text {miR-454-3p }} \times(-0.031)+\exp _{\text {miR-1271-5p }} \times(-0.094)$.

The 161 patients with early PC were divided into a low-risk group (<median PI; $n=80)$ and a high-risk group ( $\geq$ median PI; $n=81$ ). The survival time was compared between the two groups using Kaplan-Meier analysis with the log-rank test. The overall survival in the low-risk group was significantly longer than that in the high-risk group (log-rank $\mathrm{P}<0.001$; Fig. 1C). The seven-miRNA signature PI was indicated to be a promising biomarker for predicting the 2-year-survival rate of patients with PC at the early stage with an area under the ROC curve $(A U C)=0.750$ (Fig. 1D). Furthermore, as opposed to routine clinicopathological features, this seven-miRNA signature PI was a powerful independent prognostic factor, as statistical significance was retained after multivariate logistic regression analysis $[\mathrm{P}=0.002$, hazard ratio $(\mathrm{HR})=2.204$, 95\% CI: 1.285-3.196; Table II].

Targets of the seven miRNAs and pathway enrichment analysis. A total of 1,126 genes and 219 lncRNAs were predicted by using the miRNet tool based on the seven miRNAs selected (miR-424-5p, miR-139-5p, miR-5586-5p, 
Table I. Clinicopathological features of 161 patients with pancreatic at the early stage.

\begin{tabular}{lr}
\hline Characteristic & $\mathrm{n}(\%)$ \\
\hline Sex & \\
Male & $89(55.28)$ \\
Female & $72(44.72)$ \\
Age (years) & \\
$<65$ & $75(46.58)$ \\
$\geq 65$ & $86(53.42)$ \\
T-stage & \\
T1/2 & $27(16.77)$ \\
T3/4 & $134(83.23)$ \\
N-stage & \\
N1 & $42(26.09)$ \\
N0 & $115(71.43)$ \\
Nx & $4(2.48)$ \\
TNM Stage & \\
I & $19(11.80)$ \\
II & $142(88.20)$ \\
Grade & \\
G1/2 & $111(68.94)$ \\
G3/4 & $48(29.81)$ \\
Unknown & $2(1.24)$ \\
\hline
\end{tabular}

miR-126-3p, miR-3613-5p, miR-454-3p and miR-1271-5p). The miRNA-gene and lncRNA-miRNA networks were merged together and a lncRNA-miRNA-gene network was constructed using Cytoscape software (Fig. 2). Kyoto Encyclopedia of Genes and Genomes pathway enrichment analyses of the target genes were performed in miRNet using the hypergeometric test algorithm, and the top 15 pathways are presented in Fig. 3. All of the results of the pathway enrichment analysis are provided in Table SI. The analysis indicated that the target genes of seven miRNAs are involved in various pathways associated with cancer, including the 'pancreatic cancer' pathway.

Results of GSEA and the ceRNA network of the seven-miRNA signature. The GSEA was performed to identify potential associated biological pathways affected by the seven-miRNA signature. The extracellular matrix (ECM) receptor interaction pathway (Fig. 4A) and transforming growth factor (TGF)- $\beta$ signaling pathway (Fig. 4B) were significantly enriched in the high-risk group, suggesting that the seven-miRNA signature may affect the prognosis by targeting those genes involved in the two pathways. A total of 69 core enrichment genes were provided by the GSEA, eight of which were shared among the 1,126 target genes of these seven miRNAs (Fig. 4C, upper panel). A total of 34 PC-associated lncRNAs were obtained from the lncRNADisease database, six of which were shared among the 219 target lncRNAs of these seven miRNAs (Fig. 4C, lower panel). According to miRNet, there is no record of PC-associated lncRNA targeting by miR-5586-5p or miR-3613-5p. The ceRNA network contained 16 edges and 19 nodes (five miRNAs, eight genes and six lncRNAs) (Fig. 5). Gene laminin subunit $\gamma 1$ (LAMC1) and integrin subunit $\alpha 2$ (ITGA2) were involved in the ECM receptor interaction pathway. Rho associated coiled-coil containing protein kinase (ROCK)2, ROCK1, activin A receptor type 2A (ACVR2A), SMAD specific E3 ubiquitin protein ligase 1, ACVR1 and TGF- $\beta$ receptor 2 (TGFBR2) were involved in the TGF- $\beta$ signaling pathway.

\section{Discussion}

Only $<20 \%$ PCs are diagnosed at the early stages due to its asymptomatic nature (3), however, $>50 \%$ of early PC cases encounter recurrences within 12 months after surgery (4). Thanks to TCGA, large samples of early PCs can be obtained for this present study, which attempted to provide novel information on the mechanism on early PC pathogenesis. Previous studies have indicated that certain miRNAs are promising diagnostic and prognostic markers for PC $(7,29,30)$. Given the development of high-throughput sequencing technologies, an increasing number of miRNAs has been identified in recent years. Furthermore, therapies based on miRNAs hold promise to revolutionize PC treatment due to their increased efficacy compared to conventional chemoradiation-based therapies (31). Therefore, it may be worthwhile to further explore prognostic miRNA biomarkers for PC. In the present study, a novel seven-miRNA prognostic signature for predicting prognosis of early PC was obtained by comprehensively analyzing the mature miRNA expression profiles and clinical information in TCGA database using a Bioinformatics method. The seven miRNAs (miR-424-5p, miR-139-5p, miR-5586-5p, miR-126-3p, miR-3613-5p, miR-454-3p and miR-1271-5p) were obtained using LASSO and the Cox regression method. Based on the seven-miRNA signature, high-risk individuals were successfully identified among patients with early PC. In clinical practice, such high-risk individuals may be followed up more frequently. Furthermore, treatments for patients with early PC remain limited (32) and high-risk individuals have a poorer prognosis; therefore, high-risk individuals are encouraged to receive more aggressive management than the low-risk individuals, including participation in a clinical trial.

A number of previous studies have proposed various pre-miRNA-based signatures for predicting the prognosis of patients with PC (7,33-36). Dou et al (36) proposed an eight-miRNA signature (miR-1301, miR-598, miR-1180, miR-155, miR-496, miR-203, miR-193b and miR-135b), Liang et al (33) proposed a five-miRNA signature (miR-1301, miR-125a, miR-376c, miR-328 and miR-376b) and Yu et al (34) proposed a two-miRNA signature (miR-424 and miR-126). It is worth noting that the data of only several cases of PC with advanced stage (III/IV) disease are contained in TCGA; however, none of the previous studies removed these samples to focus on early-stage PC. In the present study, mature miRNA expression profiles were used instead of pre-miRNA to establish a novel miRNA-based prognostic signature for early-stage PC. The seven-miRNA signature PI is a promising biomarker for predicting 2-year-survival rate of early PC with an $\mathrm{AUC}=0.750$. Several previous studies did not provide data from ROC analyzes $(33,34,36)$. In contrast to routine 
Table II. Univariate/multivariate analysis of routine clinicopathological features and the PI of the seven-microRNA signature.

Univariate analysis

\begin{tabular}{|c|c|c|c|c|c|c|}
\hline \multirow[b]{2}{*}{ Characteristics } & \multicolumn{3}{|c|}{ Univariate analysis } & \multicolumn{3}{|c|}{ Multivariate analysis } \\
\hline & P-value & HR & $\mathrm{HR}(95 \% \mathrm{CI})$ & P-value & HR & $\mathrm{HR}(95 \% \mathrm{CI})$ \\
\hline Sex (male/female) & 0.538 & 1.141 & $0.750-1.735$ & & & \\
\hline Age $(\geq 65$ y/<65 y) & 0.076 & 1.473 & $0.960-2.259$ & & & \\
\hline $\mathrm{T}(\mathrm{T} 2 / \mathrm{T} 1)$ & 0.019 & 2.217 & $1.142-4.304$ & 0.428 & 1.597 & $0.502-5.082$ \\
\hline N (N1/N0) & 0.010 & 2.056 & $1.192-3.547$ & 0.062 & 1.948 & $0.966-3.927$ \\
\hline Stage (II/I) & 0.036 & 2.305 & $1.056-5.032$ & 0.540 & 0.621 & $0.136-2.845$ \\
\hline Grade (G3-4/G1-2) & 0.089 & 1.469 & $0.943-2.289$ & & & \\
\hline PI (high risk/low risk) & 0.000 & 2.420 & $1.548-3.782$ & 0.002 & 2.024 & $1.285-3.186$ \\
\hline
\end{tabular}

HR, hazard ratio; PI, prognostic index.

Multivariate analysis

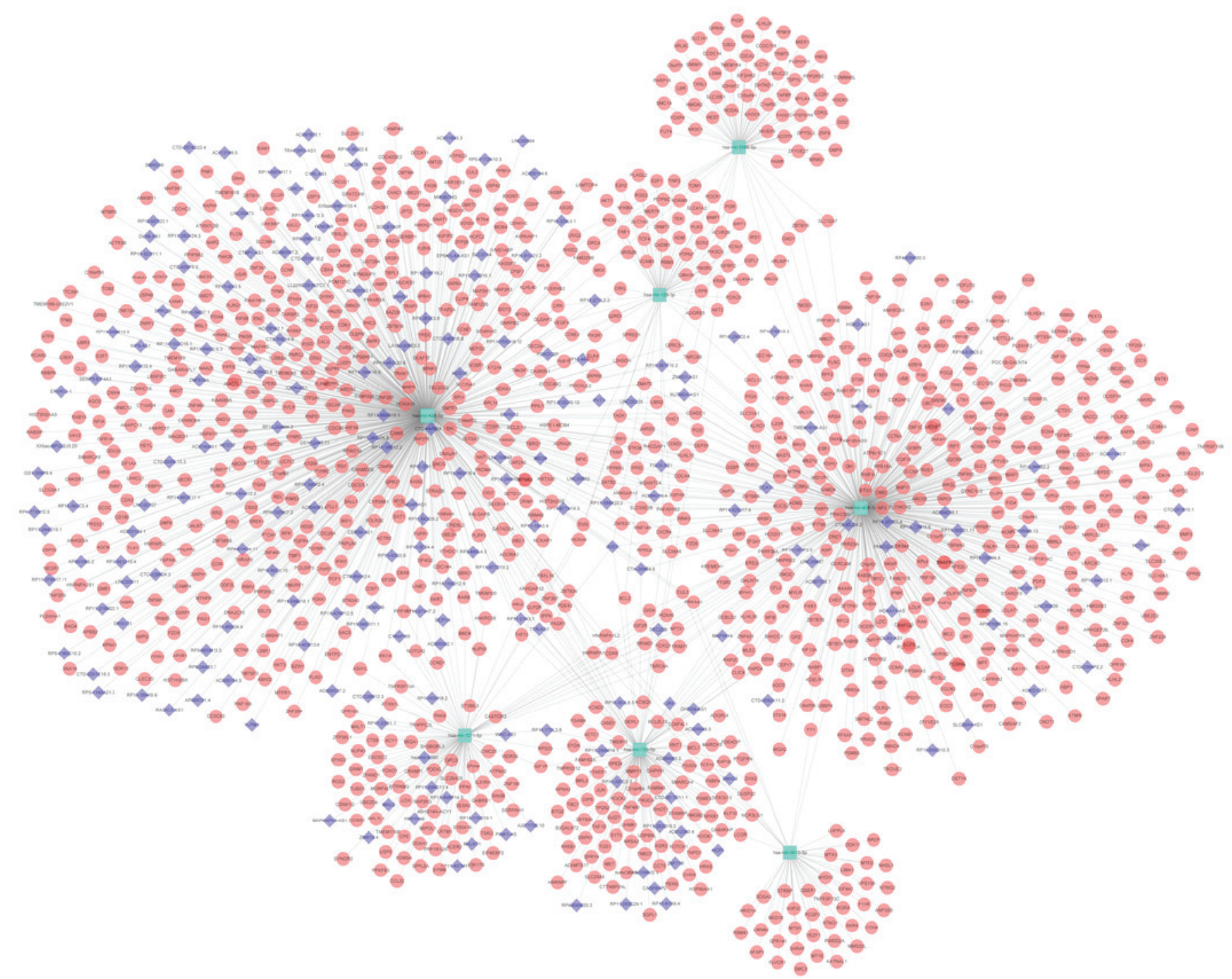

Figure 2. LncRNA-miRNA-gene network constructed using miRNet based on a seven-miRNA signature. Red dots indicate genes, blue diamonds indicate lncRNAs and green hexagons indicate miRNAs. miRNA/miR, microRNA; lncRNA, long non-coding RNA; hsa, Homo sapiens.

clinicopathological features, the seven-miRNA signature provided in the present study is an independent prognostic factor.
In the present study, GSEA was also performed and the potential ceRNA network of the seven miRNAs was constructed to explore their ultimate downstream effects. The 


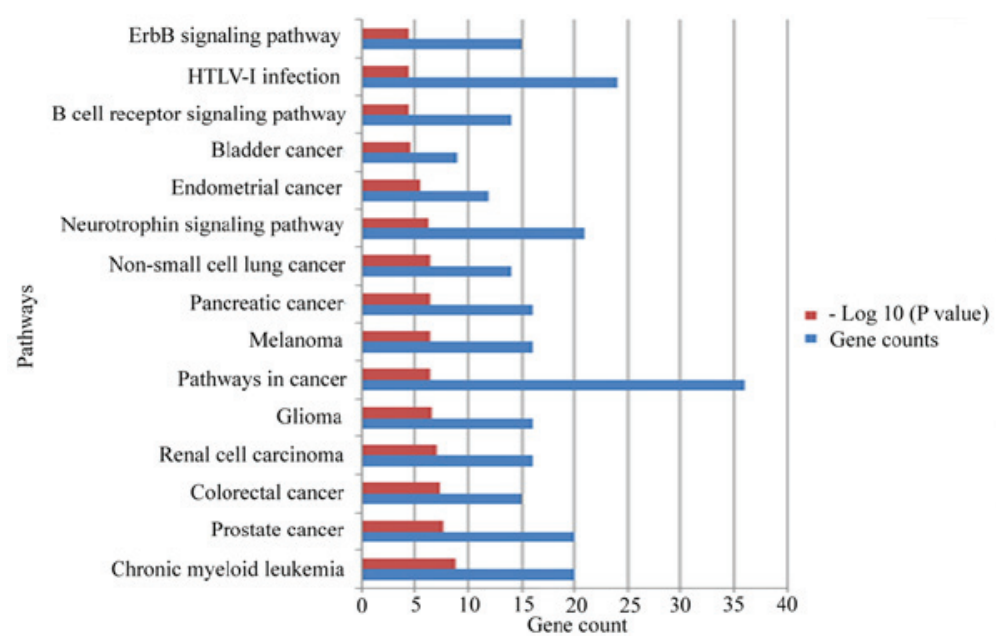

Figure 3. Top 15 significantly enriched Kyoto Encyclopedia of Genes and Genomes pathways of the seven-microRNA signature target genes.
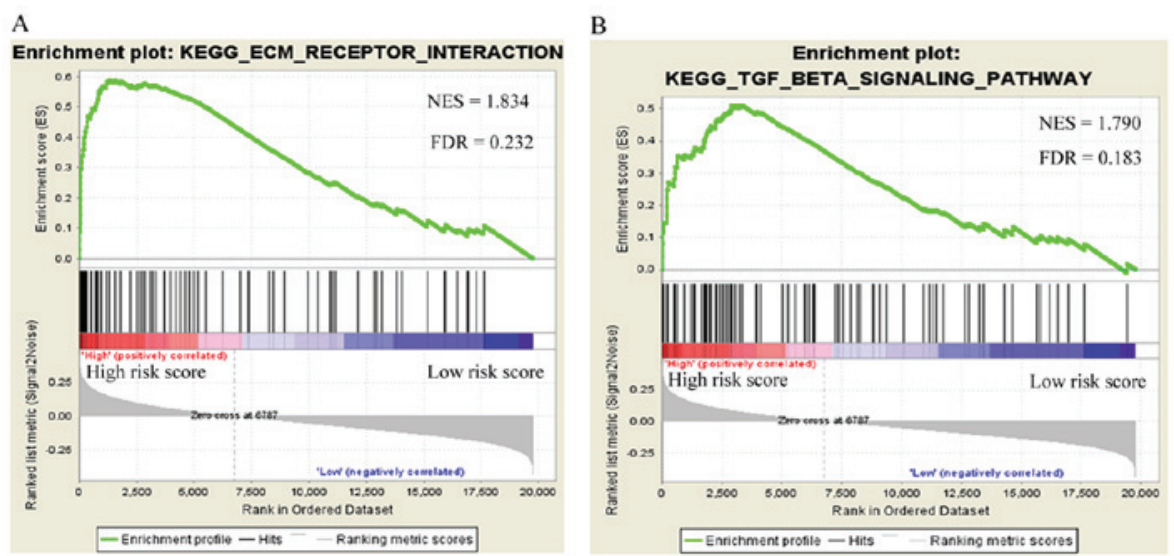

C
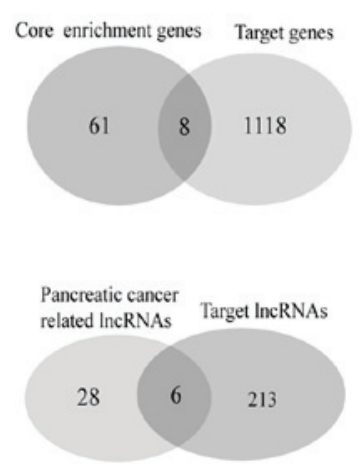

Figure 4. Results of the gene set enrichment analysis indicating that (A) the ECM receptor interaction pathway and (B) the TGF- $\beta$ signaling pathway were enriched in the high-risk group. (C) Venn analysis of common genes between target genes and core enrichment genes, and common lncRNAs between target lncRNAs and pancreatic cancer-associated lncRNAs. IncRNA, long non-coding RNA; KEGG, Kyoto Encyclopedia of Genes and Genomes; FDR, false discovery rate; NES, normalized enrichment score; ECM, extracellular matrix; TGF, transforming growth factor.

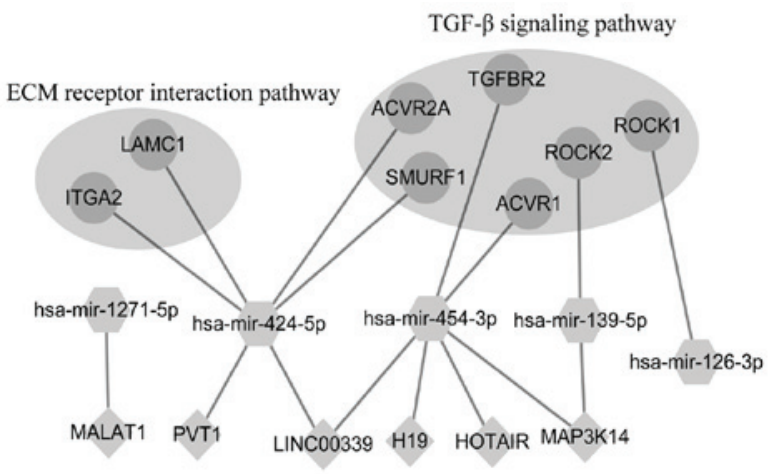

Figure 5. Predicted competing endogenous RNA network of the seven-miRNA signature. Dots indicate genes, diamonds indicate lncRNAs and hexagons indicate miRNAs. ECM, extracellular matrix; TGF, transforming growth factor; IncRNA, long non-coding RNA; miRNA/miR, microRNA; hsa, Homo sapiens; MAP3K14, mitogen-activated protein kinase kinase kinase 14; MALAT1, Metastasis Associated Lung Adenocarcinoma Transcript 1; PVT1, Pvt1 oncogene; LINC00339, long intergenic non-protein coding RNA 339; H19, H19 imprinted maternally expressed transcript; HOTAIR, HOX transcript antisense RNA; ROCK, Rho associated coiled-coil containing protein kinase; ACVR, activin A receptor; ITGA2, integrin subunit $\alpha 2$; LAMC1, laminin subunit $\gamma 1$; SMURF1, SMAD specific E3 ubiquitin protein ligase 1; TGFBR2, transforming growth factor- $\beta$ receptor 2 . potential ceRNA network comprised a total of 1,126 genes and 219 lncRNAs as predicted by using the miRNet tool, and this network provided a large number of interactions between these seven miRNAs and other genes. This may provide a valuable resource of information, as miRNA-based therapy is a promising treatment strategy for PC (37). The TGF- $\beta$ signaling pathway has been reported to facilitate the genesis of PC (38), and the present study suggested that this may be associated with downregulation of miR-454-3p, miR-139-5p and miR-126-3p. It has been reported that miR-424-5p (39) and miR-139-5p (40) are associated with PC. TGFBR2 is one of the most frequently altered genes in patients with PC (41), and the present study suggested that it may be targeted by miR-454-3p. HOTAIR has been reported to be a negative prognostic factor with an oncogenic role in PC (42), and the present study indicated that it may promote the development of PC by upregulating TGFBR2 and ACVR1 via adsorbing miR-454-3p. The ECM receptor interaction pathway has been reported to be involved in the development of various cancer types, including lung cancer (43), bladder cancer (44) and breast cancer (45). The present study suggests that miR-424-5p is downregulated by adsorption of PVTI and LINC00339, leading to upregulation of 
ITGA2 and LAMC1 involved in the ECM receptor interaction pathway to promote $\mathrm{PC}$. This suggests that the ceRNA network constructed in the present study warrants further exploration to expand the understanding of the pathogenesis of PC.

To the best of our knowledge, the present study was the first to not only propose a novel seven-miRNA prognostic signature for early PC, but also to provide their potential ceRNA network. However, the study has certain limitations. There was a lack of validation, as well as absence of prospective follow-up data from other databases or clinical trials. It is essential to validate and even improve this seven-miRNA signature in a larger independent cohort. Particularly, due to most cases of PC are diagnosed at late stage, the findings derived from the present study would need to be validated on late-stage PC samples. In addition, although a potential ceRNA network was constructed, the exact roles of these seven miRNAs in PC require further assessment; it remains elusive whether these miRNAs are causal or are merely prognostic markers for early PC.

In conclusion, a seven-miRNA expression-based prognostic signature for predicting the prognosis of patients with $\mathrm{PC}$ at the early stage was proposed in the present study. Using this signature, patients with early PC may be divided into high-risk and low-risk groups. The corresponding potential ceRNA network of the seven-miRNA expression-based prognostic signature was preliminarily explored.

\section{Acknowledgements}

Not applicable.

\section{Funding}

This study was supported by the Youth Science Foundation of Guangxi Medical University (grant no. GXMUYSF 201716).

\section{Availability of data and materials}

The datasets used and/or analyzed during the present study are available from the corresponding authors on reasonable request.

\section{Authors' contributions}

LH and YL conceived and designed the study. XB and DL performed data analyses and prepared the manuscript. YL contributed significantly to the data analyses and manuscript revision. All authors read and approved the final manuscript.

\section{Ethics approval and consent to participate}

Not applicable.

\section{Patient consent for publication}

Not applicable.

\section{Competing interests}

The authors declare that they have no competing interests.

\section{References}

1. Bray F, Ferlay J, Soerjomataram I, Siegel RL, Torre LA and Jemal A: Global cancer statistics 2018: GLOBOCAN estimates of incidence and mortality worldwide for 36 cancers in 185 countries. CA Cancer J Clin 68: 394-424, 2018.

2. Rahib L, Smith BD, Aizenberg R, Rosenzweig AB, Fleshman JM and Matrisian LM: Projecting cancer incidence and deaths to 2030: The unexpected burden of thyroid, liver, and pancreas cancers in the United States. Cancer Res 74: 2913-2921, 2014.

3. Wagner M, Redaelli C, Lietz M, Seiler CA, Friess H and Buchler MW: Curative resection is the single most important factor determining outcome in patients with pancreatic adenocarcinoma. Br J Surg 91: 586-594, 2004.

4. Yadav D and Lowenfels AB: The epidemiology of pancreatitis and pancreatic cancer. Gastroenterology 144: 1252-1261, 2013.

5. Beger HG, Thorab FC, Liu Z, Harada N and Rau BM: Pathogenesis and treatment of neoplastic diseases of the papilla of Vater: Kausch-Whipple procedure with lymph node dissection in cancer of the papilla of Vater. J Hepatobiliary Pancreat Surg 11: 232-238, 2004.

6. Bartel DP: MicroRNAs: Genomics, biogenesis, mechanism, and function. Cell 116: 281-297, 2004.

7. Schultz NA, Andersen KK, Roslind A, Willenbrock H, Wøjdemann M and Johansen JS: Prognostic microRNAs in cancer tissue from patients operated for pancreatic cancer-five microRNAs in a prognostic index. World J Surg 36: 2699-2707, 2012.

8. Yu L, Xiang L, Feng J, Li B, Zhou Z, Li J, Lin Y, Lv Y, Zou D, Lei Z and Zhang J: miRNA-21 and miRNA-223 expression signature as a predictor for lymph node metastasis, distant metastasis and survival in kidney renal clear cell carcinoma. J Cancer 9: 3651-3659, 2018.

9. Lin Y, Lv Y, Liang R, Yuan C, Zhang J, He D, Zheng X and Zhang J: Four-miRNA signature as a prognostic tool for lung adenocarcinoma. Onco Targets Ther 11: 29-36, 2018.

10. Wiemer EA: The role of microRNAs in cancer: No small matter. Eur J Cancer 43: 1529-1544, 2007.

11. Tay Y, Rinn J and Pandolfi PP: The multilayered complexity of ceRNA crosstalk and competition. Nature 505: 344-352, 2014.

12. Bak RO and Mikkelsen JG: miRNA sponges: Soaking up miRNAs for regulation of gene expression. Wiley Interdiscip Rev RNA 5: 317-333, 2014

13. Salmena L, Poliseno L, Tay Y, Kats L and Pandolfi PP: A ceRNA hypothesis: The Rosetta Stone of a hidden RNA language? Cell 146: 353-358, 2011.

14. Duell EJ, Lujan-Barroso L, Sala N, Deitz McElyea S, Overvad K, Tjonneland A, Olsen A, Weiderpass E, Busund LT, Moi L, et al: Plasma microRNAs as biomarkers of pancreatic cancer risk in a prospective cohort study. Int J Cancer 141: 905-915, 2017.

15. Namkung J, Kwon W, Choi Y, Yi SG, Han S, Kang MJ, Kim SW, Park T and Jang JY: Molecular subtypes of pancreatic cancer based on miRNA expression profiles have independent prognostic value. J Gastroenterol Hepatol 31: 1160-1167, 2016.

16. Lee KH, Lee JK, Choi DW, Do IG, Sohn I, Jang KT, Jung SH, Heo JS, Choi SH and Lee KT: Postoperative prognosis prediction of pancreatic cancer with seven microRNAs. Pancreas 44: 764-768, 2015

17. Goldman M, Craft B, Hastie M, Repečka K, Kamath A, McDade F, Rogers D, Brooks AN, Zhu J and Haussler D: The UCSC Xena platform for public and private cancer genomics data visualization and interpretation. bioRxiv: 326470, 2019.

18. Amin MB, Greene FL, Edge SB, Compton CC, Gershenwald JE, Brookland RK, Meyer L, Gress DM, Byrd DR and Winchester DP: The Eighth Edition AJCC Cancer Staging Manual: Continuing to build a bridge from a population-based to a more 'personalized' approach to cancer staging. CA Cancer J Clin 67: 93-99, 2017.

19. Ritchie ME, Phipson B, Wu D, Hu Y, Law CW, Shi W and Smyth GK: Limma powers differential expression analyses for RNA-sequencing and microarray studies. Nucleic Acids Res 43: e47, 2015 .

20. Xie M, Lv Y, Liu Z, Zhang J, Liang C, Liao X, Liang R, Lin Y and $\mathrm{Li} \mathrm{Y}$ : Identification and validation of a four-miRNA (miRNA-21-5p, miRNA-9-5p, miR-149-5p, and miRNA-30b-5p) prognosis signature in clear cell renal cell carcinoma. Cancer Manag Res 10: 5759-5766, 2018.

21. Wu TT, Chen YF, Hastie T, Sobel E and Lange K: Genome-wide association analysis by lasso penalized logistic regression. Bioinformatics 25: 714-721, 2009.

22. Tibshirani R: The lasso method for variable selection in the Cox model. Stat Med 16: 385-395, 1997. 
23. Lin Z, Cai YJ, Chen RC, Chen BC, Zhao L, Xu SH, Wang XD, Song M, Wu JM, Wang YQ, et al: A microRNA expression profile for vascular invasion can predict overall survival in hepatocellular carcinoma. Clin Chim Acta 469: 171-179, 2017.

24. Heagerty PJ, Lumley T and Pepe MS: Time-dependent ROC curves for censored survival data and a diagnostic marker. Biometrics 56: 337-344, 2000.

25. Fan Y, Siklenka K, Arora SK, Ribeiro P, Kimmins S and Xia J: miRNet-dissecting miRNA-target interactions and functional associations through network-based visual analysis. Nucleic Acids Res 44: W135-W141, 2016.

26. Demchak B, Hull T, Reich M, Liefeld T, Smoot M, Ideker T and Mesirov JP: Cytoscape: The network visualization tool for GenomeSpace workflows. F1000Res 3: 151, 2014.

27. Subramanian A, Tamayo P, Mootha VK, Mukherjee S, Ebert BL Gillette MA, Paulovich A, Pomeroy SL, Golub TR, Lander ES and Mesirov JP: Gene set enrichment analysis: A knowledge-based approach for interpreting genome-wide expression profiles. Proc Natl Acad Sci USA 102: 15545-15550, 2005.

28. Chen G, Wang Z, Wang D, Qiu C, Liu M, Chen X, Zhang Q, Yan G and Cui Q: LncRNADisease: A database for long-non-coding RNA-associated diseases. Nucleic Acids Res 41: D983-D986, 2013.

29. Tavano F, di Mola FF, Piepoli A, Panza A, Copetti M, Burbaci FP, Latiano T, Pellegrini F, Maiello E, Andriulli A and di Sebastiano P: Changes in miR-143 and miR-21 expression and clinicopathological correlations in pancreatic cancers. Pancreas 41: 1280-1284, 2012.

30. Giovannetti E, van der Velde A, Funel N, Vasile E, Perrone V, Leon LG, De Lio N, Avan A, Caponi S, Pollina LE, et al: High-throughput microRNA (miRNAs) arrays unravel the prognostic role of MiR-211 in pancreatic cancer. PLoS One 7: e49145, 2012.

31. Gurbuz N and Ozpolat B: MicroRNA-based targeted therapeutics in pancreatic cancer. Anticancer Res 39: 529-532, 2019.

32. Chiaravalli M, Reni M and O'Reilly EM: Pancreatic ductal adenocarcinoma: State-of-the-art 2017 and new therapeutic strategies. Cancer Treat Rev 60: 32-43, 2017.

33. Liang L, Wei DM, Li JJ, Luo DZ, Chen G, Dang YW and Cai XY: Prognostic microRNAs and their potential molecular mechanism in pancreatic cancer: A study based on The Cancer Genome Atlas and bioinformatics investigation. Mol Med Rep 17: 939-951, 2018.

34. Yu Y, Feng $\mathrm{X}$ and Cang S: A two-microRNA signature as a diagnostic and prognostic marker of pancreatic adenocarcinoma Cancer Manag Res 10: 1507-1515, 2018.
35. Zhou X, Huang Z, Xu L, Zhu M, Zhang L, Zhang H, Wang X, Li H, Zhu W, Shu Y and Liu P: A panel of 13-miRNA signature as a potential biomarker for predicting survival in pancreatic cancer. Oncotarget 7: 69616-69624, 2016.

36. Dou D, Yang S, Lin Y and Zhang J: An eight-miRNA signature expression-based risk scoring system for prediction of survival in pancreatic adenocarcinoma. Cancer Biomark 23: 79-93, 2018.

37. PaiP,RachaganiS,Are Cand Batra SK: Prospects of miRNA-based therapy for pancreatic cancer. Curr Drug Targets 14: 1101-1109, 2013.

38. Principe DR, DeCant B, Mascariñas E, Wayne EA, Diaz AM, Akagi N, Hwang R, Pasche B, Dawson DW, Fang D, et al: TGF $\beta$ signaling in the pancreatic tumor microenvironment promotes fibrosis and immune evasion to facilitate tumorigenesis. Cancer Res 76: 2525-2539, 2016.

39. Wu K, Hu G, He X, Zhou P, Li J, He B and Sun W: MicroRNA-424-5p suppresses the expression of SOCS6 in pancreatic cancer. Pathol Oncol Res 19: 739-748, 2013.

40. Ma J, Zhang J, Weng YC and Wang JC: EZH2-mediated microRNA-139-5p regulates epithelial-mesenchymal transition and lymph node metastasis of pancreatic cancer. Mol Cells 41: 868-880, 2018.

41. Ku JL, Yoon KA, Kim WH, Jang Y, Suh KS, Kim SW, Park YH and Park JG: Establishment and characterization of four human pancreatic carcinoma cell lines. Genetic alterations in the TGFBR2 gene but not in the MADH4 gene. Cell Tissue Res 308: 205-214, 2002.

42. Kim K, Jutooru I, Chadalapaka G, Johnson G, Frank J, Burghardt R, Kim S and Safe S: HOTAIR is a negative prognostic factor and exhibits pro-oncogenic activity in pancreatic cancer. Oncogene 32: 1616-1625, 2013.

43. Li D, Yang W, Zhang Y, Yang JY, Guan R, Xu D and Yang MQ: Genomic analyses based on pulmonary adenocarcinoma in situ reveal early lung cancer signature. BMC Med Genomics 11 (Suppl 5): S106, 2018.

44. Gao X, Chen Y, Chen M, Wang S, Wen X and Zhang S: Identification of key candidate genes and biological pathways in bladder cancer. PeerJ 6: e6036, 2018.

45. Yeh MH, Tzeng YJ, Fu TY, You JJ, Chang HT, Ger LP and Tsai KW: Extracellular matrix-receptor interaction signaling genes associated with inferior breast cancer survival. Anticancer Res 38: 4593-4605, 2018.

This work is licensed under a Creative Commons

Attribution-NonCommercial-NoDerivatives 4.0 International (CC BY-NC-ND 4.0) License. 